\title{
Neuromyelitis optica spectrum disorder following COVID-19 infection with increase in pre-existing anti-aquaporin-4 antibodies
}

\author{
Alexandre Jentzer ${ }^{1} \cdot$ Clarisse Carra-Dallière $^{2} \cdot$ Claire Lozano $^{1} \cdot$ Sophie Riviere ${ }^{3} \cdot$ Olivier Darmon $^{3} \cdot$ Xavier Ayrignac $^{2}$. \\ Pierre Labauge $^{2} \cdot$ Thierry Vincent $^{1}$ (D)
}

Received: 23 December 2021 / Revised: 12 January 2022 / Accepted: 13 January 2022 / Published online: 22 January 2022

(c) The Author(s), under exclusive licence to Springer-Verlag GmbH Germany 2022

\section{Dear Sirs,}

Neuromyelitis optica (NMO) is a severe inflammatory disease of the central nervous system (CNS) characterized by simultaneous or consecutive attacks of acute optic neuritis $(\mathrm{ON})$ and transverse myelitis (TM). Unlike multiple sclerosis, NMO is associated in most patients with the presence of specific and pathogenic auto-antibodies targeting aquaporin-4 (AQP4), the most abundant water channel protein in the CNS [1]. More recently, the notion of neuromyelitis optica spectrum disorder (NMOSD) was introduced to include patients presenting, at least initially, with isolated $\mathrm{ON}$ or TM or, more rarely, brainstem or brain inflammation [2]. It is well known that severe respiratory syndromecoronavirus-2 (SARS-CoV-2) infection can be associated with symptomatic involvement of the central or peripheral nervous system [3]. Among associated CNS demyelination, encephalitis/encephalomyelitis are the most common presentations. Longitudinally extensive transverse myelitis (LETM) are more rarely reported [4]. Causality between SARS-CoV-2 infection and CNS demyelination is not fully established and pathogenesis is not completely understood. We describe for the first time a patient with pre-existing asymptomatic anti-AQP4 antibodies who developed severe LETM following coronavirus disease 2019 (COVID-19) infection associated with a strong increase in anti-AQP4

Thierry Vincent

t-vincent@chu-montpellier.fr

1 Department of Immunology, Saint Eloi Montpellier University Hospital, Montpellier University, 80 Avenue Augustin FLICHE, 34 295, cedex 5 Montpellier, France

2 Department of Neurology, Gui de Chauliac Montpellier University Hospital, Montpellier University, Montpellier, France

3 Department of Internal Medicine, Saint Eloi Montpellier University Hospital, Montpellier University, Montpellier, France antibody titers in the serum suggesting a direct relationship between viral infection and the autoimmune-mediated demyelination.

The patient, a 71-year-old Caucasian woman, was previously followed for Hereditary Hemorrhagic Telangiectasia (HHT) diagnosed in 2018 with no history of demyelination. She had recurrent episodes of hepatic encephalopathy, secondary to HHT hepatic arterio-venous shunt. In October 2020, she had uncomplicated SARS-CoV-2 infection with positive nasal swab reverse-transcription polymerase chain reaction (RT-PCR). Three months later, during an episode of hepatic encephalopathy, she developed a subacute paraplegia. Spinal cord MRI revealed an extensive myelitis from C7 to T6 (Fig. 1a, b), with bright spotty lesions on axial T2-weighted images (Fig. 1c). The diagnosis of NMOSD was confirmed by detection of anti-AQP4 antibodies in the patient's serum by indirect immunofluorescence (IIF) on primate cerebellum slides. Serum concentration was determined using a semi-quantitative commercially available cellbased assay (CBA; Euroimmun, Lübeck, Germany) and confirmed with a more sensitive and accurate home-made CBA using living cells and flow cytometry as previously described [5]. We also determined complement-mediated cytotoxicity of anti-AQP4 antibodies using AQP4 expressing HEK-293 T transduced cells as previously described [6]. We took advantage of the blood samples drawn during the follow-up of the patient for her history of HHT to compare anti-AQP4 antibodies before and after the COVID-19 episode. As shown in Fig. 1d, anti-AQP4 antibodies were already present in the serum drawn in 2019, 11 months before SARS-CoV-2 infection and more than 1 year before LETM. Serum concentration strongly increased following SARS-CoV-2 infection $(p<0.001)$ and was associated with a similar increase of the complement-mediated cytotoxicity measured in the different post-COVID-19 sera $\left(R^{2}=0.83, p=0.044\right)$. Surprisingly, when analyzed by IIF on brain and cerebellum slides, sera drawn before and after COVID-19 infection gave 

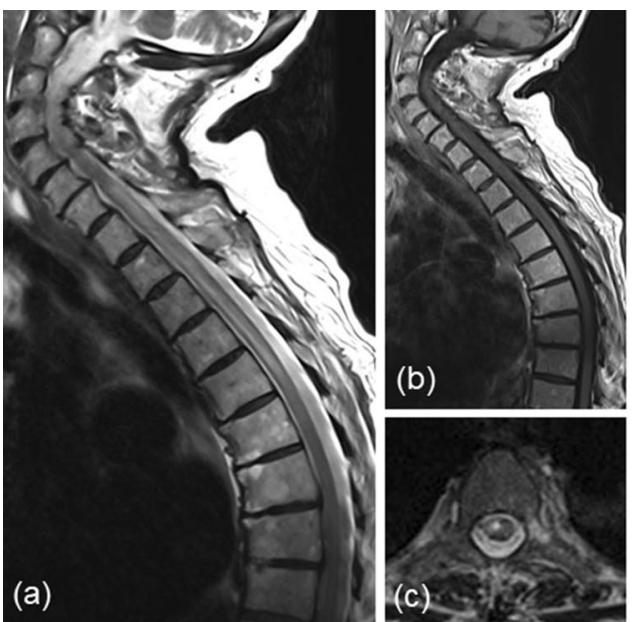

(d)

Fig. 1 Extensive myelitis following SARS-CoV2 infection in a patient previously followed for Hereditary Hemorrhagic Telangiectasia (HHT). Spinal cord MRI revealed an extensive myelitis from C7 to T6 on T2-weighted sagittal image (a), without gadolinium enhancement on contrast-enhanced T1-weighted sequence (b). A bright spotty lesion, very specific of anti-AQP4 positive NMOSD, was observed on T2-weighted axial images (c). (d) Anti-AQP4 serum concentration and complement-mediated serum cytotoxicity were measured in sera obtained before and after COVID-19 infection with cell-based assays using AQP4 expressing human HEK-293 T cells as previously described [6]. Anti-AQP4 concentration was measured by

rise to different pattern of fluorescence. Indeed, whereas we observed with all post-infection sera a typical anti-AQP4 staining of the pia (Fig. 2c) and the perivascular VirchowRobin spaces (Fig. 2d), the serum from 2019 produced linear and radial fluorescence in the molecular layer (Fig. 2a) and around vessels (Fig. 2b).

Angiotensin-converting enzyme 2 (ACE2), the cell surface molecule recognized by the spike glycoprotein of SARS-CoV-2 virus, is expressed in the CNS [7]. Nevertheless, the pathogenesis of CNS demyelination episodes observed after COVID-19 infection is not well understood. Various hypotheses were proposed including molecular mimicry between viral and neurological antigens, immune dysregulation through bystander activation or systemic inflammation, increased blood-brain barrier (BBB) permeability allowing antibody entry in the CNS, hypoxia, and/or a direct viral pathogenic effect [8]. Interestingly, the patient referred in this case report already presented anti-AQP4 antibodies at least 11 months before the COVID-19 infection and more than 1 year before neurological symptoms. SARS-CoV-2 infection was associated with an important increase in antibody concentration in the serum. Anti-AQP4 antibody concentration was not assessed in the CSF, but it is likely that systemic inflammation induced by the virus with secretion of inflammatory cytokines such as interleukin-1 and 6 may have increased BBB permeability, thus favoring antibody entry

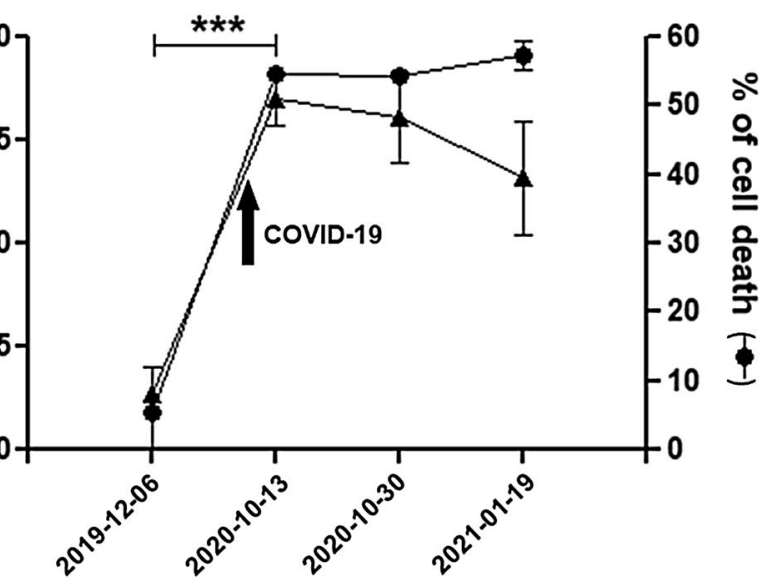

flow cytometry after incubation with patient's sera and donkey antiHuman IgG conjugated with Alexa-Fluor 647 as secondary antibody. The mean fluorescence intensity (MFI) is represented in left axis (black triangles). Complement-mediated cytotoxicity was measured after incubation with heat inactivated patient's sera and rabbit complement. The percentage of dead cells was quantified using 7AAD labeling and is represented on the right axis (black circles). All sera were tested in triplicate and means \pm SD are shown. The black arrow represents SARS-CoV2 infection. A one-way ANOVA test with Bonferroni correction was used to compare sera before and after COVID$19(* * * p<0.001)$

in the CNS. COVID-19-associated inflammation may also have induced post-translational modifications of antiAQP4 antibodies such as altered glycosylation that may have increased their effector functions and amplified their pathogenic potential $[9,10]$. Nevertheless, the strong correlation observed between antibody concentration and the complement-mediated cytotoxic activity measured in the different sera drawn before and after SARS-CoV-2 infection does not plead for this hypothesis (Fig. 1d). Interestingly, the observed pattern of fluorescence on the cerebellum slides by IIF was different between sera drawn before and after COVID-19 infection with linear and radial fluorescence within the molecular layer and around vessels before infection and a typical anti-AQP4 staining of the pia and the Virchow-Robin spaces in the brain and the cerebellum with all sera drawn after SARS-CoV-2 infection (Fig. 2). These data may indicate a phenomenon of epitope spreading induced by the SARS-CoV-2 infection with auto-antibodies recognizing different parts and/or isoforms of the AQP4 molecule.

In conclusion, we describe for the first time to our knowledge a case of SARS-CoV-2 infection with subsequent development of NMOSD in a patient with preexisting asymptomatic anti-AQP4 antibodies. Although causality cannot be definitively established, SARS-CoV-2 infection was associated with a strong increase in antiAQP4 antibody concentration and complement-mediated 

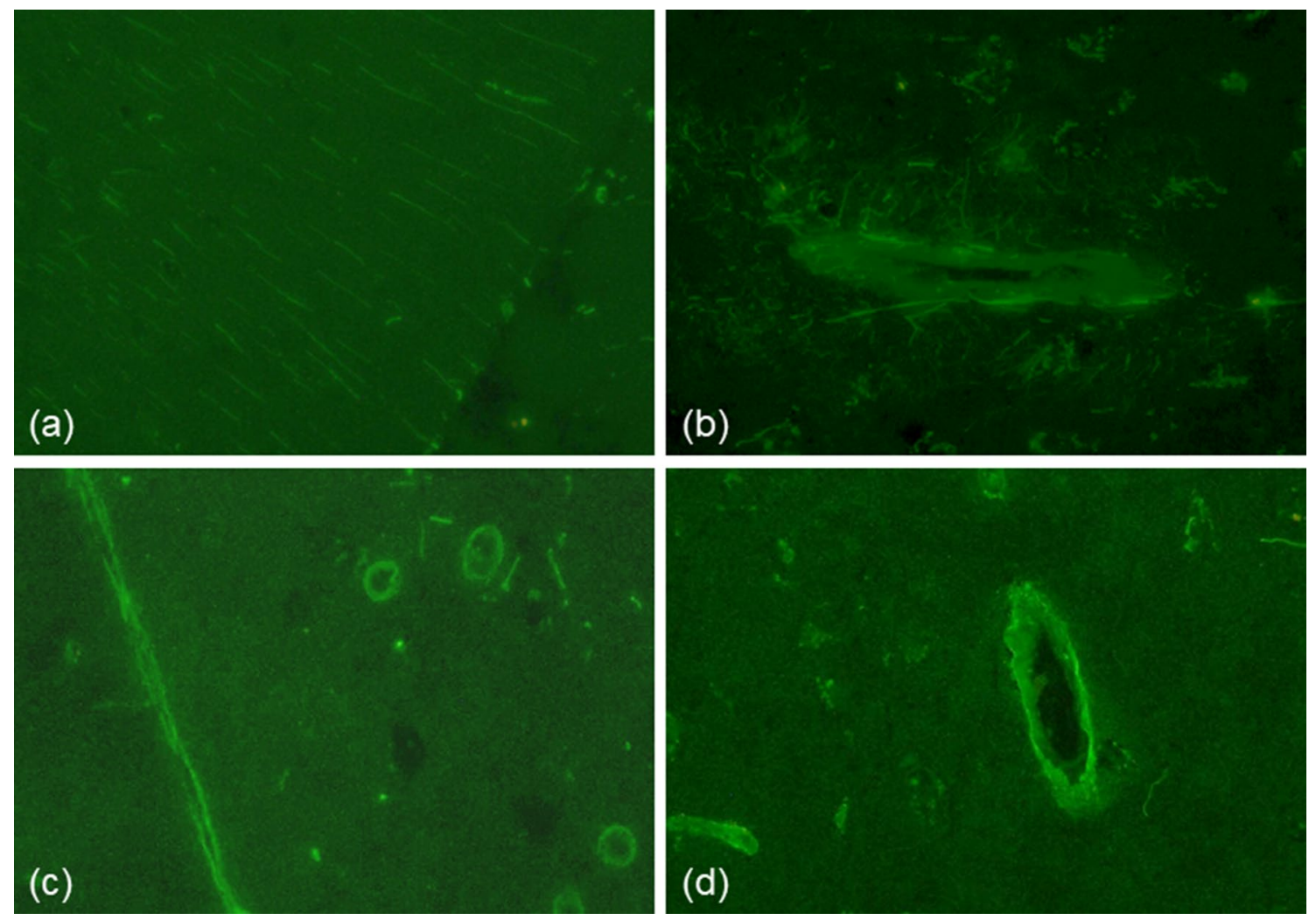

Fig. 2 Different pattern of immunofluorescence on brain and cerebellum slides before and after SARS-Cov-2 infection. Primate cerebellum and brain sections (Werfen, Barcelona, Spain) were used for immunofluorescence analysis with diluted sera drawn before (2019) or after (2020 and 2021) the COVID-19 episode according to the manufacturer's instructions. With the serum from 2019, we observed

cytotoxicity of the patient's serum against AQP4 expressing cells, suggesting a direct relationship between viral infection, immune dysregulation, and demyelination.

Author contributions Thierry Vincent did literature review and drafted the manuscript. Alexandre Jentzer did experiments and data curation. Alexandre Jentzer, Clarisse Carra-Dallière, Claire Lozano, Sophie Riviere, Olivier Darmon, Xavier Ayrignac, and Pierre Labauge have critically revised and finalized the manuscript. All authors have approved the final version of the manuscript.

Funding The authors received no financial support for the research, authorship, and publication of this article.

\section{Declarations}

Conflicts of interest The authors declared no potential conflict of interest with respect to the research, authorship, and publication of this article.

Ethical approval This study was approved by our local ethics committee. a linear and radial fluorescence within the molecular layer of the cerebellum (a) and around the vessels of the brain parenchyma (b). All sera drawn after the SARS-CoV-2 infection produced typical immunofluorescence staining of anti-AQP4 antibodies with linear staining of the pia (c), and the perivascular Virchow-Robin spaces (d)

Informed consent Informed consent was obtained from the patient included in this study.

\section{References}

1. Lennon VA, Kryzer TJ, Pittock SJ, Verkman AS, Hinson SR (2005) IgG marker of optic-spinal multiple sclerosis binds to the aquaporin-4 water channel. J Exp Med 202:473-477

2. Jarius S, Paul F, Weinshenker BG, Levy M, Kim HJ, Wildemann B (2020) Neuromyelitis optica. Nat Rev Dis Primers 6:85

3. Mao L, Jin H, Wang M, Hu Y, Chen S, He Q, Chang J, Hong C, Zhou Y, Wang D, Miao X, Li Y, Hu B (2020) Neurologic manifestations of hospitalized patients with coronavirus disease 2019 in Wuhan, China. JAMA Neurol 77:683-690

4. Ismail II, Salama S (2021) Association of CNS demyelination and COVID-19 infection: an updated systematic review. J Neurol. https://doi.org/10.1007/s00415-021-10752-x

5. De Vidi I, Boursier G, Delouche N, Portales P, Cadars E, Bouthier M, Mettling C, Lin YL, Thouvenot E, Carlander B, Camu W, Antel JP, Bar-Or A, Zephir H, Vermersch P, De Seze J, Corbeau P, Eliaou JF, Vincent T (2011) Strategy for anti-aquaporin-4 autoantibody identification and quantification using a new cell-based assay. Clin Immunol 138:239-246 
6. Chanson JB, Alame M, Collongues N, Blanc F, Fleury M, Rudolf G, de Seze J, Vincent T (2013) Evaluation of clinical interest of anti-aquaporin-4 autoantibody followup in neuromyelitis optica. Clin Dev Immunol 2013:146219

7. Wu Y, Xu X, Chen Z, Duan J, Hashimoto K, Yang L, Liu C, Yang C (2020) Nervous system involvement after infection with COVID-19 and other coronaviruses. Brain Behav Immun $87: 18-22$

8. Koga M, Takahashi T, Kawai M, Fujihara K, Kanda T (2011) A serological analysis of viral and bacterial infections associated with neuromyelitis optica. J Neurol Sci 300:19-22
9. Chakraborty S, Gonzalez J, Edwards K, Mallajosyula V, Buzzanco AS, Sherwood R, Buffone C, Kathale N, Providenza S, Xie MM, Andrews JR, Blish CA, Singh U, Dugan H, Wilson PC, Pham TD, Boyd SD, Nadeau KC, Pinsky BA, Zhang S, Memoli MJ, Taubenberger JK, Morales T, Schapiro JM, Tan GS, Jagannathan P, Wang TT (2021) Proinflammatory IgG Fc structures in patients with severe COVID-19. Nat Immunol 22:67-73

10. Goulabchand R, Vincent T, Batteux F, Eliaou JF, Guilpain P (2014) Impact of autoantibody glycosylation in autoimmune diseases. Autoimmun Rev 13:742-750 